POS PROCEEDINGS

\title{
The extragalactic sky in the Fermi era
}

\section{D.A. Sanchez on behalf of Fermi-LAT collaboration *i}

Laboratoire d'Annecy-le-Vieux de Physique des Particules, Université de Savoie, CNRS/IN2P3, France

E-mail: david.sanchez@lapp.in2p3.fr

Frontiers of Fundamental Physics 14 - FFP14,

15-18 July 2014

Aix Marseille University (AMU) Saint-Charles Campus, Marseille

\footnotetext{
*Speaker.

${ }^{\dagger}$ This work is supported by the Labex ENIGMASS
} 


\section{The Fermi Large Area Telescope}

The Fermi is a NASA satellite devoted to the observation of the $\gamma$ ray sky. Launched by a Delta-II rocket in June 2008 and flying at a low altitude orbit $(565 \mathrm{~km})$, Fermi has two instruments aboard: The Gamma-ray Burst Monitor (GBM) and the Large Area Telescope (LAT).

The LAT detects $\gamma$-rays from $20 \mathrm{MeV}$ to more than $300 \mathrm{GeV}$. In survey mode, each point of the sky is seen approximately 30 minutes every 3 hours. The LAT is composed of three sub-detectors [9]. A cartoon of the instrument is shown on Fig. 1. The LAT is made of 16 identical towers, each towers is composed of a tracker module and a calorimeter module. The tracker is a stack of silicon strips and tungsten layers allowing $i$ ) the conversion of the photon into an electron-positron pair and ii) the measurement of the track of the particles. In the calorimeter module, the CsI(Tl) bars allow to measure the energy deposit and the position of the electromagnetic shower to create an image of the latter. To reduce the charged particle background an Anti-Coincidence Detector (ACD) covers the tracker. Its design allows the rejection of incoming charged particles without vetoing the out-coming one, produced by the highest energy $\gamma$-ray induced shower.
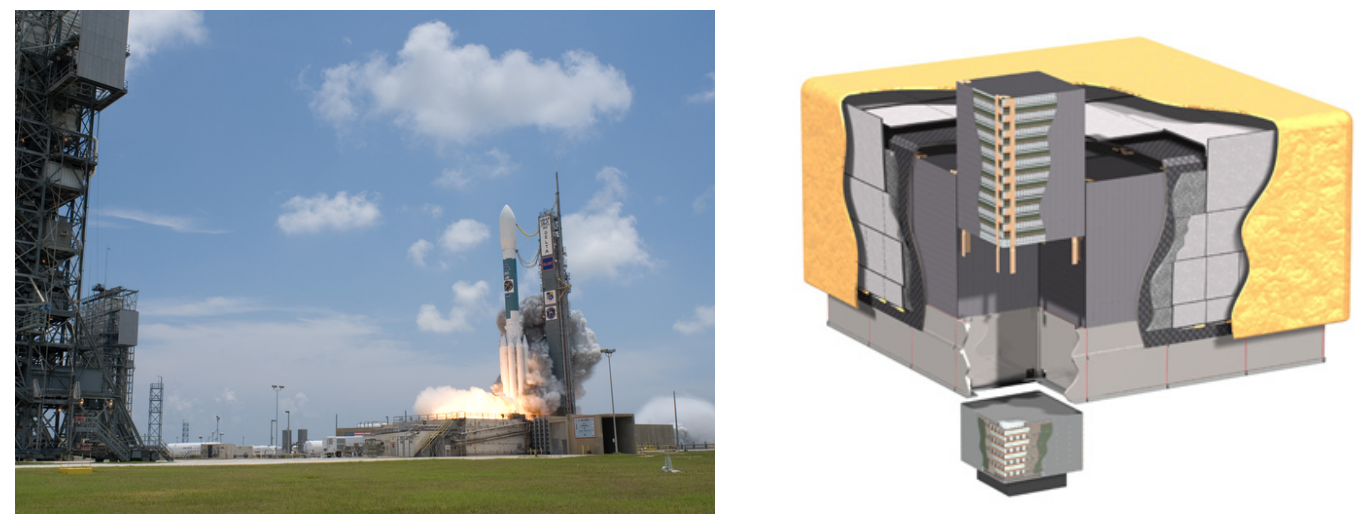

Figure 1: (left) A picture of the launch of Fermi aboard the Delta II rocket. (right) A cartoon of the LAT where a module of the tracker and the calorimeter can be seen.

\section{Focus on selected results}

This section describes a selection of few important results obtained with the Fermi-LAT.

\subsection{The Fermi-LAT sources catalogue (3FGL)}

Thanks to its all-sky monitoring capabilities, the LAT has already produced flux-limited catalogues of the $\gamma$ ray sky. Based on 4 years of data taking the third catalogue of sources (3FGL [11]) contains more than 3000 sources. An all-sky map above $1 \mathrm{GeV}$ is shown on figure 2 on which the galaxic plan and bright point-like sources are clearly visible.

Numerous improvements have been made in the data analysis with respect to previous catalogues (i.e. 1FGL and 2FGL). The last instrumental response functions (i.e. Pass 7) were used and the energy range has been increased to $100 \mathrm{MeV}-300 \mathrm{GeV}$. The improved point spread function allows a better localization of sources- especially sources with a hard spectrum. 
As in the 2FGL catalogue [13] approximately a third of the sources are unassociated (see figure 2). The majority (58\%) of sources are Active Galactic Nuclei (AGN) and most of them are blazars. The majority of blazars seen by the LAT are BL Lac objects (395 BL Lacs and 301 FSRQs) which can be an indication of different beaming patterns, different acceleration or $\gamma$-ray absorption processes.
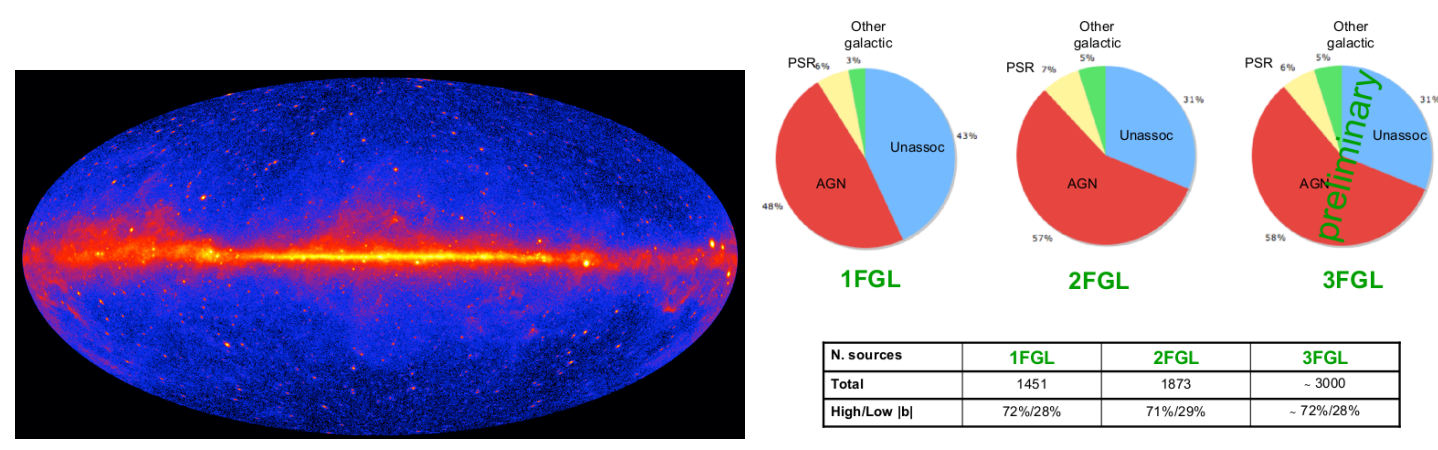

Figure 2: (left) Map in galactic coordinates above $1 \mathrm{GeV}$ of the sky with 4 years of data. Extracted from [11]. (right) Content of the Fermi sources catalogue. The 1FLG was build with 11 months, the 2FGL with 2 years and the 3FLG with 4 years. Extracted from [11].

\subsection{Variability: The Fermi All-sky Variability Analysis (FAVA)}

The Fermi collaboration has recently developed an All-sky Variability Analysis (FAVA [8]) which allow to detected variability of sources on week time-scales. This method is based on the expected number of $\gamma$-rays (computed from the past averaged emission) compared to the observed one during a week. FAVA has few interesting advantages with respect to the standard likelihood method used to analyse Fermi data:

- it is independent of any modelling of the backgrounds or of the source spectrum,

- it is not expensive in term of CPU usage and allows fast all sky survey.

The figure 3 shows the all-sky map for 2 energy bins ( $>100 \mathrm{MeV}$ and $\mathrm{E}>800 \mathrm{MeV}$ ) and for one week of data. A flaring source is seen with a positive significance while a source in quiescent state is seen with a negative significance. In 47 months, 215 flaring $\gamma$ ray sources have been found. The analysis currently runs every weeks allowing a monitoring of the variability of the sky.

\subsection{Individual sources}

Starburst galaxies Starburst galaxies present a high rate of star forming and supernovae. As an example, the supernovae rate in M 82 and NGC 253 is 10 times the rate in our Milky Way. These two starburst galaxies were the first one detected by Fermi in $\gamma$-ray [2]. The same objects have been detected in the TeV energies range by VERITAS (M82, [4]) and H.E.S.S. (NGC 253, [5]).

The formation of stars produces cosmic rays that interact with interstellar gas and radiation. This interaction produces diffuse $\gamma$-rays that can be detected. Since the detection of the firsts Starburst galaxies, a large number have been detected [7] allowing population studies. Figure 4, left panel, shows the nice linear correlation between the $\gamma$-ray luminosity and the star forming rate. 


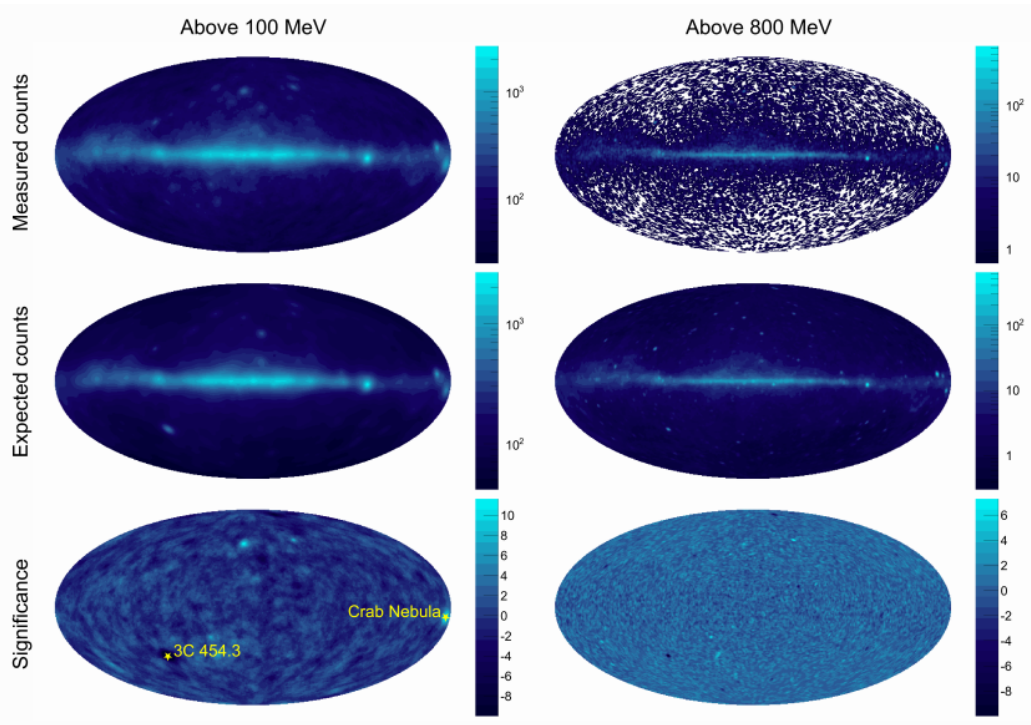

Figure 3: For energies above $100 \mathrm{MeV}$ (left) and $800 \mathrm{MeV}$ (right), this maps are, from top to bottom, the measured counts, the expected counts derived from the averaged model, and the variability significance. The data have been taken from 2009-02-02 to 2009-02-09. Extracted from [8].
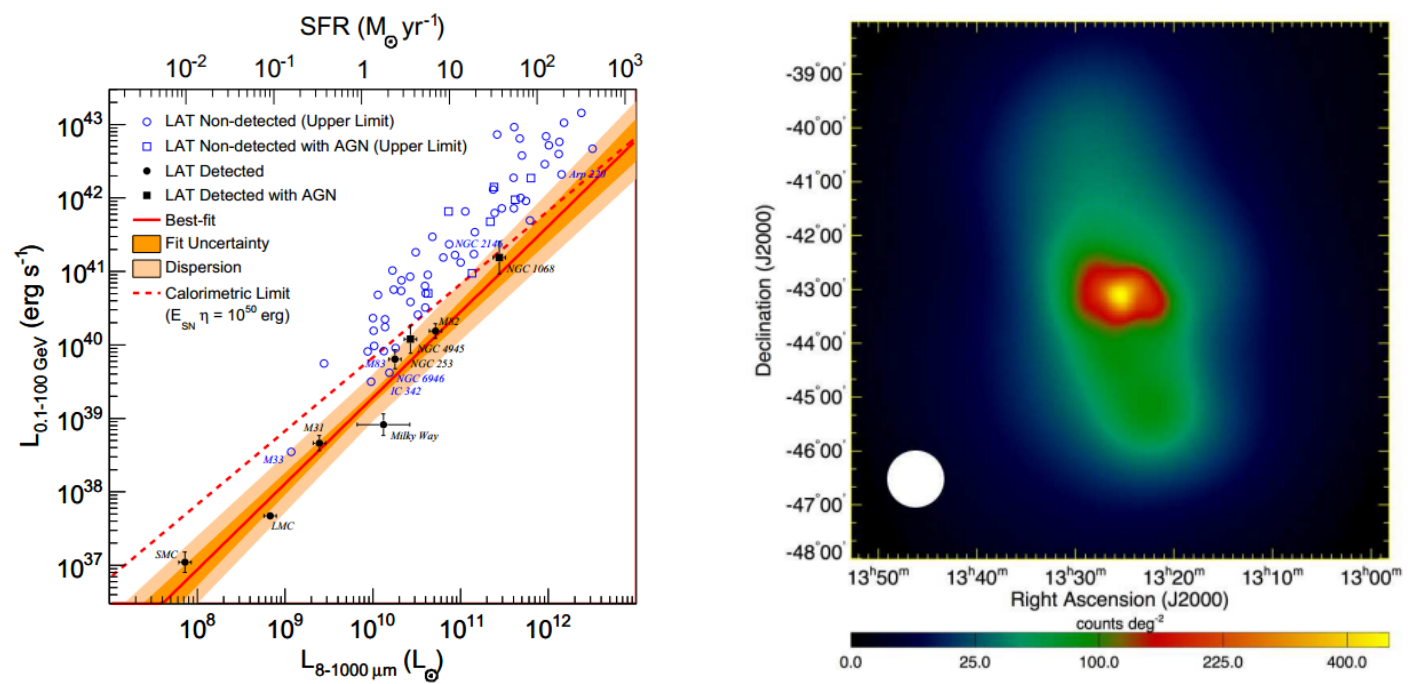

Figure 4: (Left) $\gamma$-ray luminosity $(0.1-100 \mathrm{GeV})$ as a function of the the infrared luminosity. The star forming rate is estimated from this infrared luminosity. Extracted from [7]. (Right) Count map above $200 \mathrm{MeV}$ of the lobe of the radio galaxy Centaurus A. Extracted from [1].

Centaurus A At a distance of $3800 \mathrm{kpc}$, Centaurus A is the closest Radio Galaxy. Fermi has detected an extended emission from the giant radio lobes of this object [1]. The total angular extent is approximately $10 \mathrm{deg}$ and the flux of the lobes account for more that $50 \%$ of the total flux (core+lobes).

The spatially extended emission is interpreted as inverse-Compton of energetic electrons on the cosmic microwave background. The detection of the lobes in $\gamma$-rays implies that electrons of energy of $0.1-1 \mathrm{TeV}$ have been either efficiently transported from the core to the lobes or accelerated 
in the lobes themselves.

\subsection{Measurement of the extragalactic background light}

The extragalactic background light (EBL) is one of the fundamental quantities in cosmology. The EBL is a field of infrared photons produced by the first stars and reprocessed by dust. The EBL records the entire history of the Universe. Nevertheless, foregrounds, especially the zodiacal light, prevents direct measurement of the EBL density.

While travelling to the Earth, $\gamma$-rays, produced by extragalactic sources, interact with the EBL photons to produce $\mathrm{e}^{+} \mathrm{e}^{-}$pairs. The observed spectrum is then modify and the EBL absorption increases with the photon energy. The intrinsic spectra is linked to the observed one following:

$$
\phi_{\mathrm{obs}}(E)=\phi_{\text {int }} \cdot \exp (-b \tau(E, z))
$$

where $\tau$ is the optical depth and is dependent of the energy and the redshift. Several EBL density models are available in the literature to compute $\tau$. In the following, $b$ is a factor used to account for the imprecision of the model.

The absorption is not linear with the energy and a spectral feature, named wiggle, that is energy-dependant, is present. By looking for a collective behaviour in the LAT spectra of distant blazars, the imprint of the EBL has been detected. Figure 5 shows the stacked spectra of sources lying in 3 redshift bins (0-0.2,0.2-0.5 and 0.1-1.6) [6].

This detection and measurement of the EBL show that its density is close to the level predicted by the galaxy count-usually considered as a lower limit. This method also allows to rule out models that predict too huge absorption. Following a similar analysis, the H.E.S.S. collaboration found similar and complementary results in a lower redshift band but at higher energy [3].

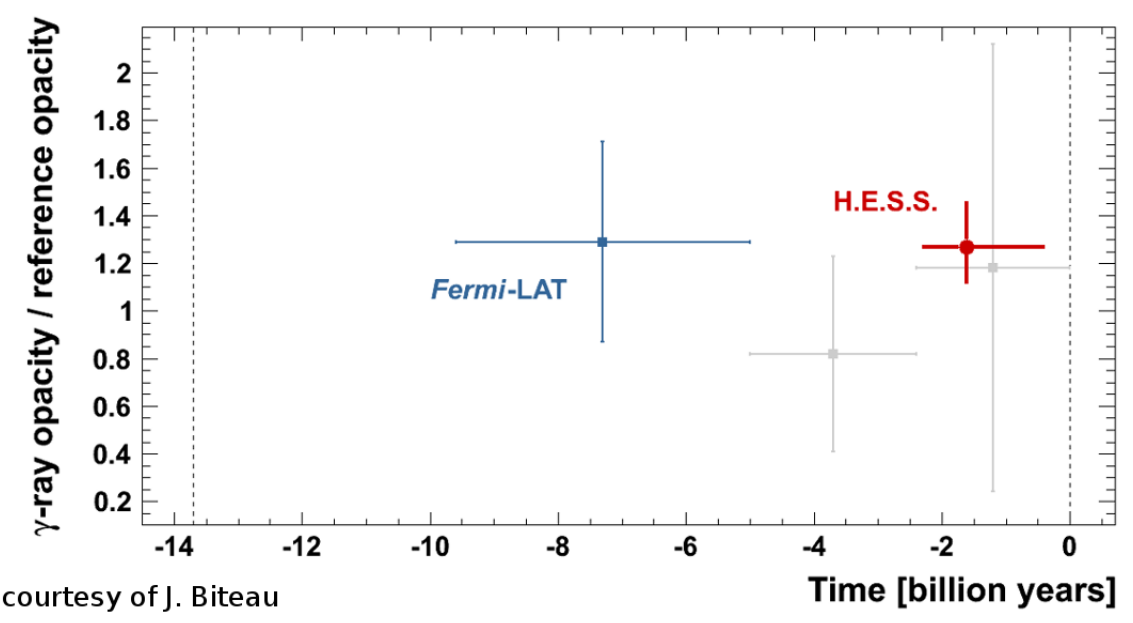

Figure 5: . Value of $b$, i.e measured opacity/ reference opacity, for the model of [12] as a function of the time in the past. The measurement presented have been made using the LAT data and H.E.S.S. data. Courtesy of J. Biteau 


\section{Prospectives}

Fermi has been launched more than 6 years ago and has exceeded it original life-time of 5 years. The LAT is performing smoothly and since no consumable is aboard, the LAT might potentially continue taking data until the spacecraft re-enter in the Earth atmosphere.

A lot of exciting discoveries were made with the LAT: Radio lobes of Centaurus A, starburst galaxies, high energy emission of Gamma-ray bursts, EBL measurement or studies of blazars populations. After 6 years, the LAT collaboration has developed the Time-domain astronomy especially with the FAVA analysis and the opportunity to probe very long time scales.

The up-coming new instrumental response functions (IRFs) named Pass 8 [10], thanks to a better knowledge of the instrument, will also improve the sensibility and increase the energy range. Figure 6 shows a comparison of the effective area of the currently used IRFs and the new Pass 8 IRFs. Pass 8 IRFs are foreseen to be available in 2015.

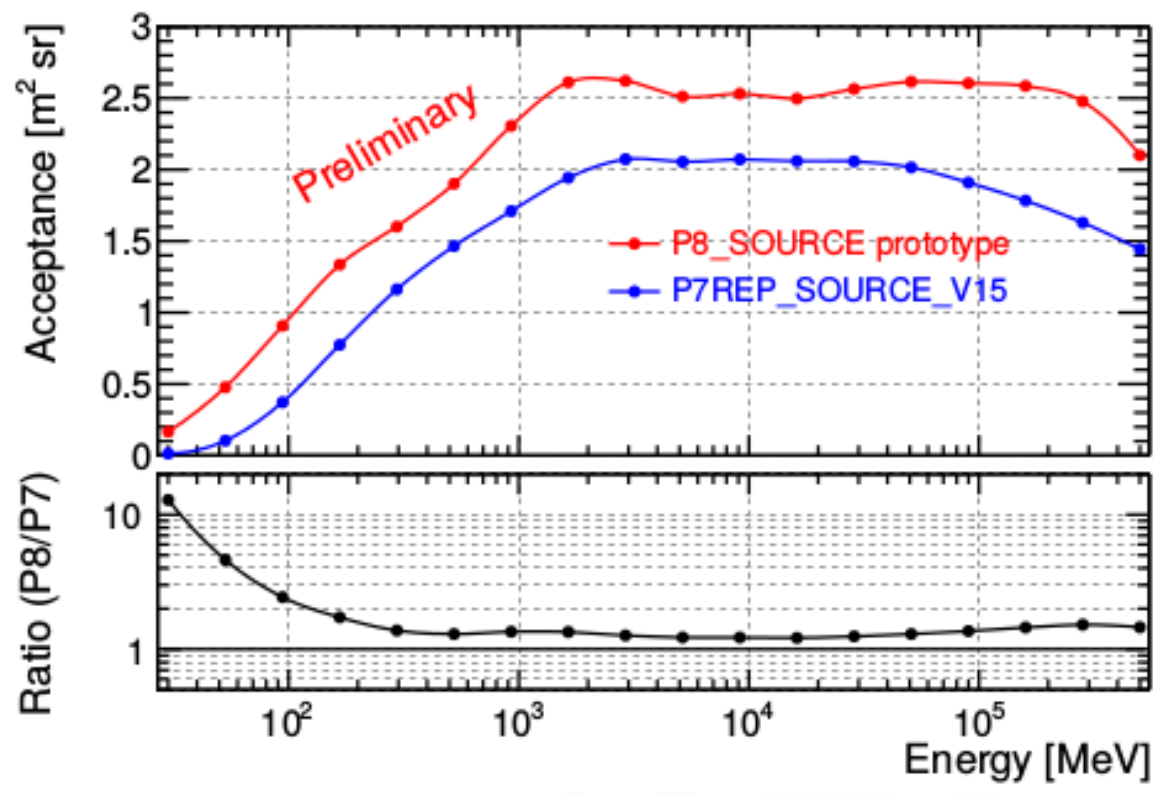

Figure 6: Top panel: Pass 8 (red) and Pass 7 (blue) effective area. The lower panel is the ratio between both. Extracted from [10].

\section{Acknoledgements}

The Fermi-LAT Collaboration acknowledges support for LAT development, operation and data analysis from NASA and DOE (United States), CEA/Irfu and IN2P3/CNRS (France), ASI and INFN (Italy), MEXT, KEK, and JAXA (Japan), and the K.A. Wallenberg Foundation, the Swedish Research Council and the National Space Board (Sweden). Science analysis support in the operations phase from INAF (Italy) and CNES (France) is also gratefully acknowledged.

\section{References}

[1] Abdo, A. A., Ackermann, M., Ajello, M., et al. 2010, Science, 328, 725 
[2] Abdo, A. A., Ackermann, M., Ajello, M., et al. 2010, ApJL, 709, L152

[3] Abramowski, A., Acero, F., et al. 2013, A\&A, 550, AA4

[4] Acciari, V. A., Aliu, E., et al. 2009, Nature, 462, 770

[5] Acero, F., Aharonian, F., Akhperjanian, A. G., et al. 2009, Science, 326, 1080

[6] Ackermann, M., Ajello, M., Allafort, A., et al. 2012, Science, 338, 1190

[7] Ackermann, M., Ajello, M., Allafort, A., et al. 2012, ApJ, 755, 164

[8] Ackermann, M., Ajello, M., Albert, A., et al. 2013, ApJ, 771, 57

[9] Atwood, B., et al. 2009, ApJ, in press, arXiv:0902.1089

[10] Bruel, P. for the Fermi collaboration, 5th Fermi symposium, 2014

[11] Cavazzuti, E. for the Fermi collaboration, 5th Fermi symposium, 2014

[12] Franceschini, A., Rodighiero, G., \& Vaccari, M. 2008, A\&A, 487, 837

[13] Nolan, P. L., Abdo, A. A., Ackermann, M., et al. 2012, ApJS, 199, 31 